\title{
Hybrid Portable Low-cost and Modular Cosmic Ray Muon and Neutron Detector
}

\section{Xiaochun He ${ }^{* a}$, Carola Butler ${ }^{a}$, Montegomery Steele ${ }^{a}$, Sawaiz Syed ${ }^{a}$, James Shirk ${ }^{a}$, Nadia Qutob $^{a}$, Emily Knutson ${ }^{a}$, Yung-Chi Jao ${ }^{a}$ and Ting-Cun Wei ${ }^{b}$}

${ }^{a}$ Georgia State University

25 Park Place, Rm 605

Atlanta, GA 30303, USA

${ }^{b}$ Northwestern Polytechnical University

No. 127 West Youyi Road, Beilin District

Xi'an 710072, Shaanxi, P.R. China

E-mail: xhe@gsu.edu

\begin{abstract}
A state-of-the-art portable, low-cost, and modular cosmic ray muon and neutron detector prototype has been developed at Georgia State University for the simultaneous measurement of cosmic ray muon and neutron flux. The detector consists of three layers of plastic scintillator and a neutron-cell with liquid scintillator mounted on an extruded aluminum frame. The scintillation light is collected through embedded wavelength shifting fibers which are coupled to silicon photomultipliers (SiPM). The data acquisition (DAQ) system of this detector consists of a Raspberry PI and a custom-made 4-channel SiPM interface board. The interface board also has a built-in GPS, accelerometer, temperature, pressure, and humidity sensors. One of the applications of this detector is to study the correlation between the flux variations and the space/earth weather at global scale by installing arrays of this detector around the world. It could also be modified for extensive air shower measurements. In the talk, we will present the details of the detector design, the DAQ system and the initial test results from this detector.
\end{abstract}

PACS: Cosmic ray, scintillator, wavelength shifting fiber, SiPM

36th International Cosmic Ray Conference -ICRC2019-

July 24th - August 1st, 2019

Madison, WI, U.S.A.

${ }^{*}$ Speaker. 


\section{Introduction}

In recent years, many interesting applications of the cosmic ray measurements have been discovered, including cosmic ray muon tomography for homeland security [1], volcanic activity monitoring, nuclear reactor core monitoring [2], and the discovery of a big void of Khufu's pyramid $[3,4]$. Numerous studies have reported correlation between the dynamical changes of the Earth's weather patterns and cosmic ray flux variations measured at sea level $[5,6,7,8,9]$. At the same time, there is a growing interest and need to monitor space radiation with cosmic ray flux measurement at the surface of earth [10,11,12].

Through a century-long effort of technological development of cosmic ray detection, especially in the astroparticle physics community, a variety of cosmic ray detectors have been developed world-wide with varying sizes, costs and technology choices.

For dynamically monitoring the earth and space weather using cosmic rays, one needs to simultaneously measure the muon and neutron flux variation near the surface of the earth at a global scale. This requires an installation of a large number of muon and neutron detectors around the world with identical acceptance and performance at a low cost. Measuring muons is relatively simple using plastic scintillator sheets, however, it is generally a challenge to measure neutrons.

A state-of-the-art portable, low-cost, and modular cosmic ray muon and neutron detector prototype has been developed at Georgia State University (GSU) for the measurement of cosmic ray muon and neutron flux variations simultaneously. The detector consists of three layers of plastic scintillator and a neutron-cell with liquid scintillator mounted on an extruded aluminum frame. The scintillation light is collected through embedded wavelength shifting fibers which are coupled to silicon photomultipliers (SiPM). The data acquisition (DAQ) system of this detector consists of a Raspberry PI and a custom-made 4-channel SiPM interface board. The interface board also has a built-in GPS, accelerometer, temperature, pressure, and humidity sensors. In this article, we present the details of the detector design, the DAQ system and the initial test results.

\section{Cosmic Ray Detector Design}

The design of the portable, low-cost cosmic ray telescope was based on the development of a hadronic calorimeter for the sPHENIX experiment at Brookhaven National Laboratory [13]. Panels of plastic scintillator $(20 \mathrm{~cm} \times 20 \mathrm{~cm} \times 1 \mathrm{~cm})$ with an embedded wavelength shifting (WLS) fiber are used for muon detection as shown in Fig. 1. The scintillation light produced by muon particles is kept in the panel by reflective foil and is absorbed by the fiber. The two ends of the fiber are brought to the corner of the panel where an SiPM readout PCB is attached.

Neutron detection is challenging since they do not carry net charge. Traditionally, one popular method of detecting fast neutrons (a few $\mathrm{MeV}$ up to $100 \mathrm{MeV}$ range) is to use a large volume of liquid scintillator, coupled with expensive high voltage photomultiplier tubes. Helium-4 has also been used in neutron detection [14]. These types of neutron detectors are not very portable and are very costly.

Given the growing popularity of using SiPM for scintillation light detection in recent years and its low cost [13], we followed this technique and developed a method of using wavelength shifting fibers to sample light in a liquid scintillator for fast neutron measurement. The fiber is encased in a 

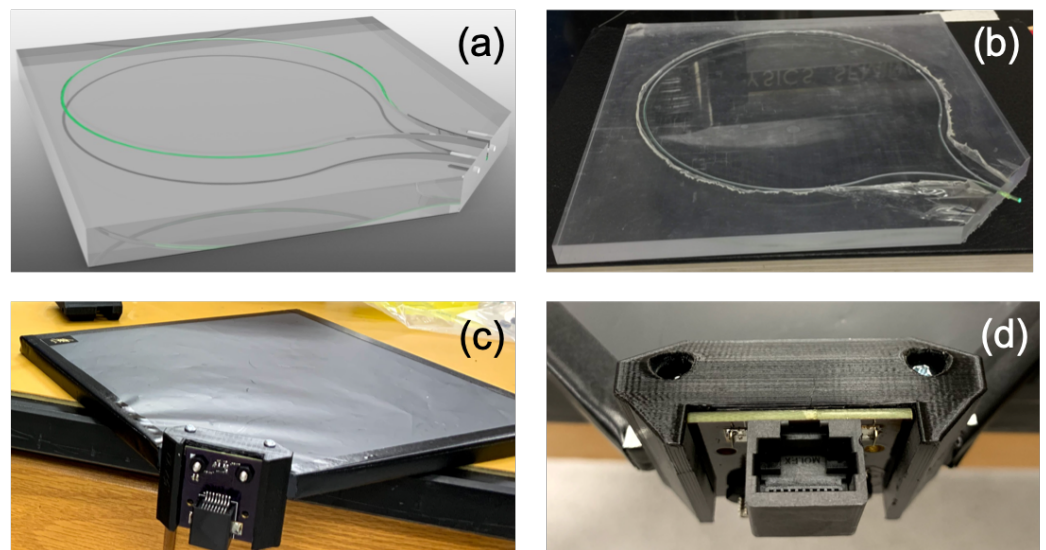

Figure 1: Scintillating panel design and assembly. (a) 3D model design for machining the panel; (b) machined panel with a wavelength shifting fiber glued in a circular groove; (c) assembled panel with a customized SiPM readout PCB; and (d) a close-up view of the PCB's RJ-45 connector.

thin glass tube filled with optical grease for index-matching. The construction of a neutron cell is shown in Fig. 2.

(a) Machined neutron cell components

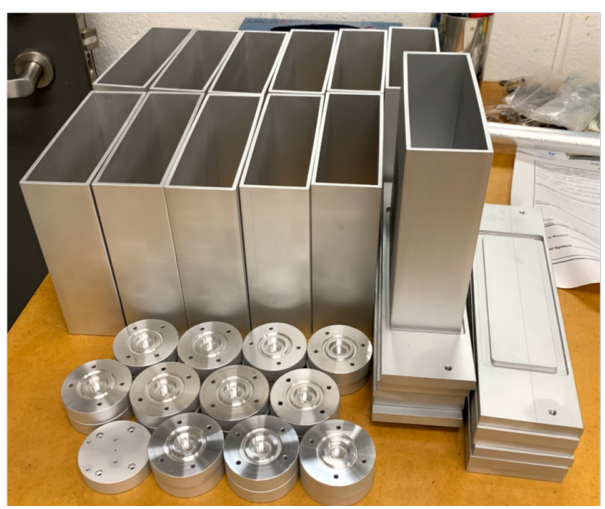

(b) Neutron cell assembly

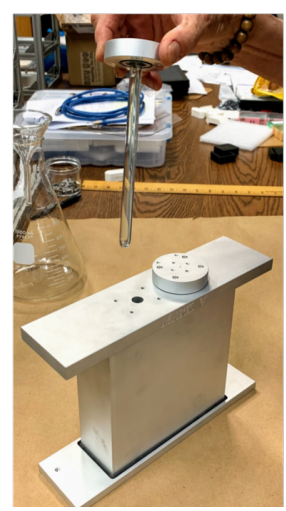

(c) Neutron in telescope

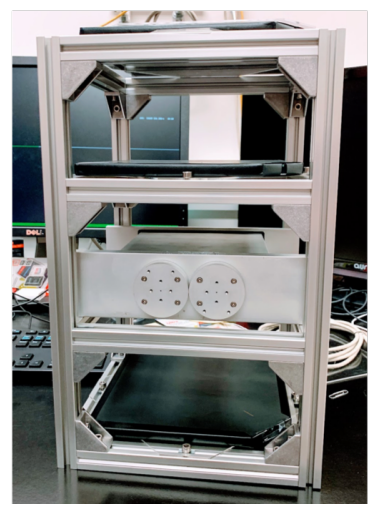

Figure 2: Neutron cell construction. (a) machined neutron cell components for 12 units; (b) neutron cell assembly. Glass tube is glued to the cap which is then screwed to the cell tank; and (c) an assembled muon/neutron telescope. No electronics are attached.

The assembly of a cosmic ray telescope is shown in Fig. 3. The overall dimension of the telescope is $24 \mathrm{~cm} \times 24 \mathrm{~cm} \times 40 \mathrm{~cm}$. Figure 3 (a) shows a muon telescope configuration. A muon/neutron telescope is shown in Fig. 3(b).

\section{Low-cost Readout}

The cosmic ray telescope readout consists of a Raspberry PI, a 4-channel SiPM interface PIHat developed at GSU as shown in Fig. 4. The scintillating signals from both the muon panels and the neutron cell are read out through a sensor PCB as shown in the left photo in Fig. 4.

The SiPM bias voltage is supplied through a daughter board which is plugged to the front side of the Raspberry Pi interface board (see Fig. 4). There are four independent channels in the current 


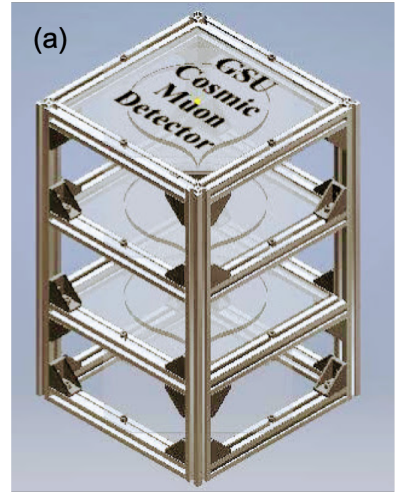

Muon telescope

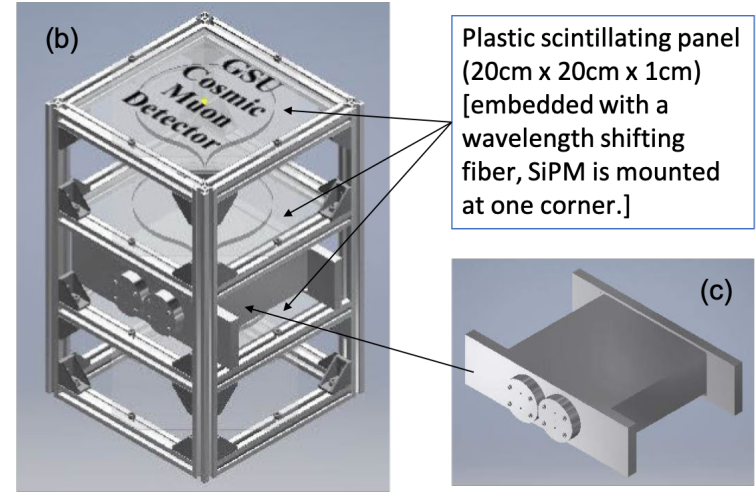

Muon/neutron telescope

Neutron cell

Figure 3: 3D model design of the cosmic ray telescope: (a) muon telescope which consists of three plastic scintillating panels; (b) muon/neutron telescope which consists of three plastic scintillating panels and one neutron cell sandwiched by two lower panels; and (c) neutron cell model.

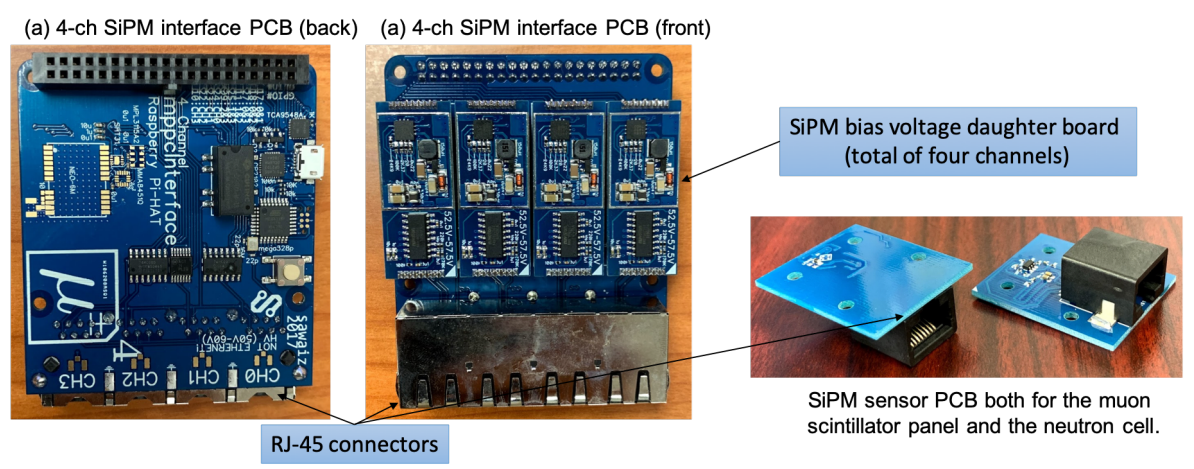

Figure 4: Raspberry PI interface board for reading out SiPM sensors.

design which can be set via a software control running on the Raspberry PI. The details of the SiPM bias voltage board can be found in Fig. 5. Figure 6 shows coincidence counts between paired panels as a function of the applied bias voltage for optimizing the SiPM bias voltage setting. As it is shown in Fig. 6, the coincidence counts level off when the bias voltage is above 54.0 volts.

\section{GEANT4-based Monte Carlo Simulation}

A GEANT4-based [15] simulation of the cosmic ray telescope has also been developed to determine the detector acceptance and efficiency. Figure 7 shows a muon only telescope detector display. A combined muon and neutron telescope simulation is under development. Also shown in Fig. 7 are tracks from 200 muon particles at $2.0 \mathrm{GeV} / \mathrm{c}$.

\section{Test Results}

We have performed a few quick tests of the cosmic ray muon telescope. Figure 8 shows the test setup and the scope traces of signals from the telescope. 

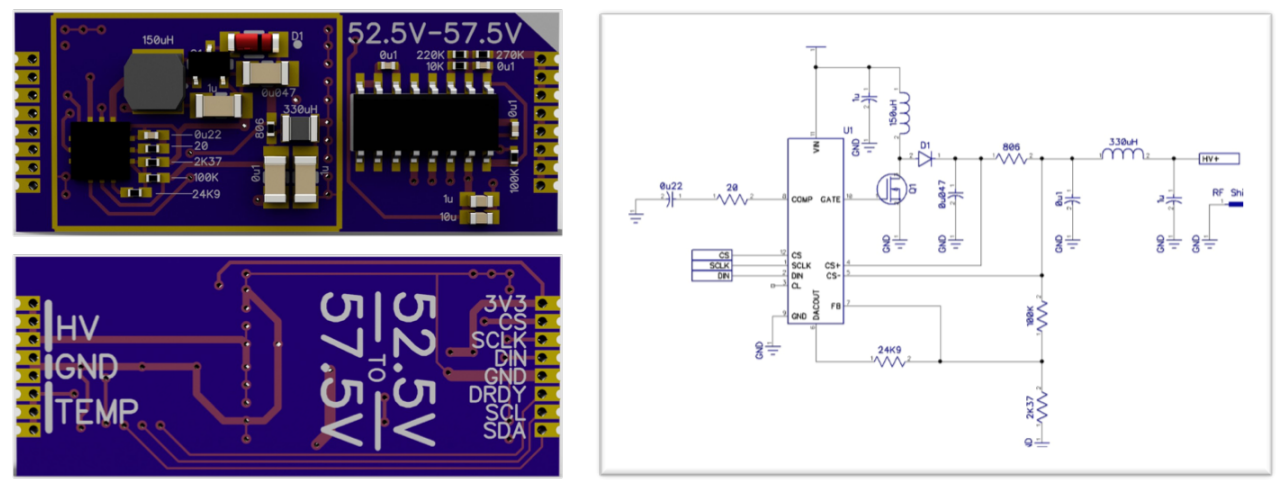

Figure 5: SiPM bias voltage board.

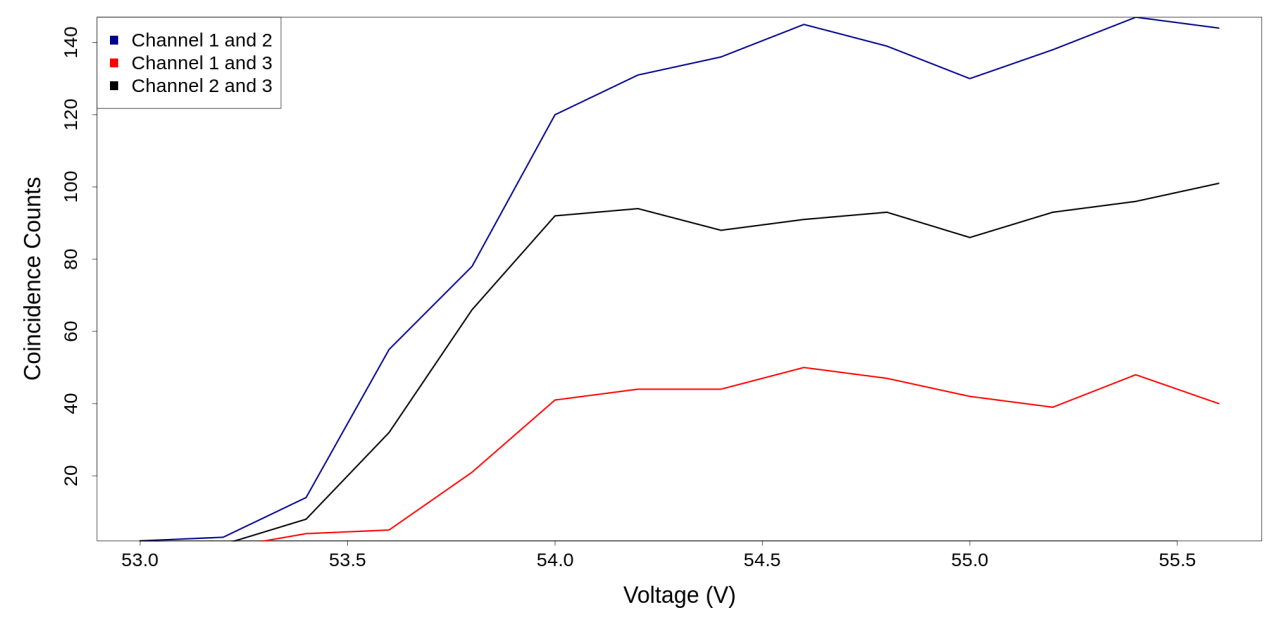

Figure 6: Optimizing the SiPM bias voltage settings using the coincidence counts of paired panels.

The data was recorded every minute for about six days on the second floor of a 5-floor building at Georgia State University, Atlanta. The data consists of the coincident counts between each pair of panels and the counts from each panel. Figure 9 shows the coincidence counts of the top two panels as a function of time. The blue data points represent the 10-minute (top) and 60-minute (bottom) running average, respectively. The minute-count is very consistent with the expectation of the average muon counts at sea level $\left(\sim 1 \mathrm{~cm}^{-2} \mathrm{~min}^{-1}\right.$ for horizontal detector [16]). Since each panel is $20 \mathrm{~cm} \times 20 \mathrm{~cm} \times 1 \mathrm{~cm}$, one would expect 400 counts per minute on average without the shielding of the building floors. By requiring the coincidence of two panels $14 \mathrm{~cm}$ apart on top of four floors of concrete shielding, our telescope recorded approximately 120 counts per minute.

Other tests including neutron cell efficiency and telescope consistencies are currently underway.

\section{Summary and Outlook}

A portable, low-cost cosmic ray telescope has been successfully developed at Georgia State University. We are currently constructing 30 telescope units which will be distributed at select 


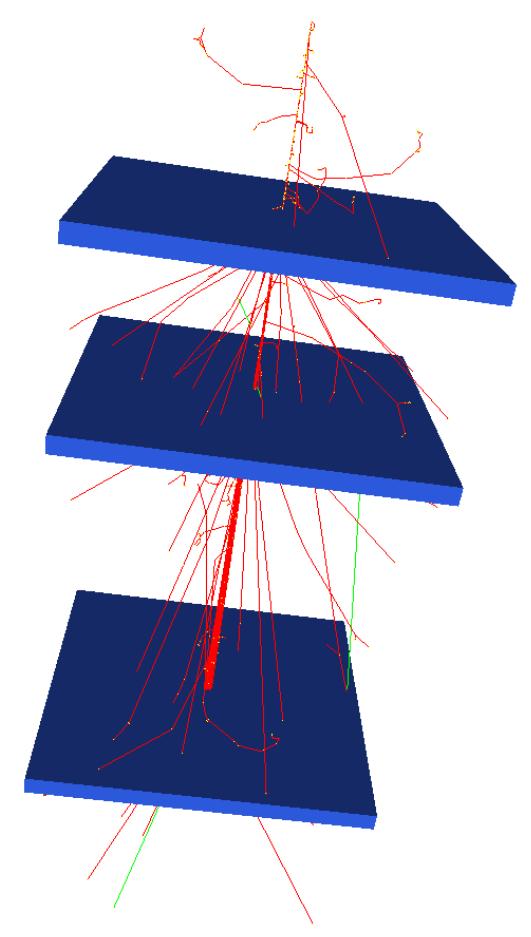

Figure 7: GEANT4-based simulation of the cosmic ray muon telescope with 200 muon events at $2.0 \mathrm{GeV} / \mathrm{c}$ in momentum.
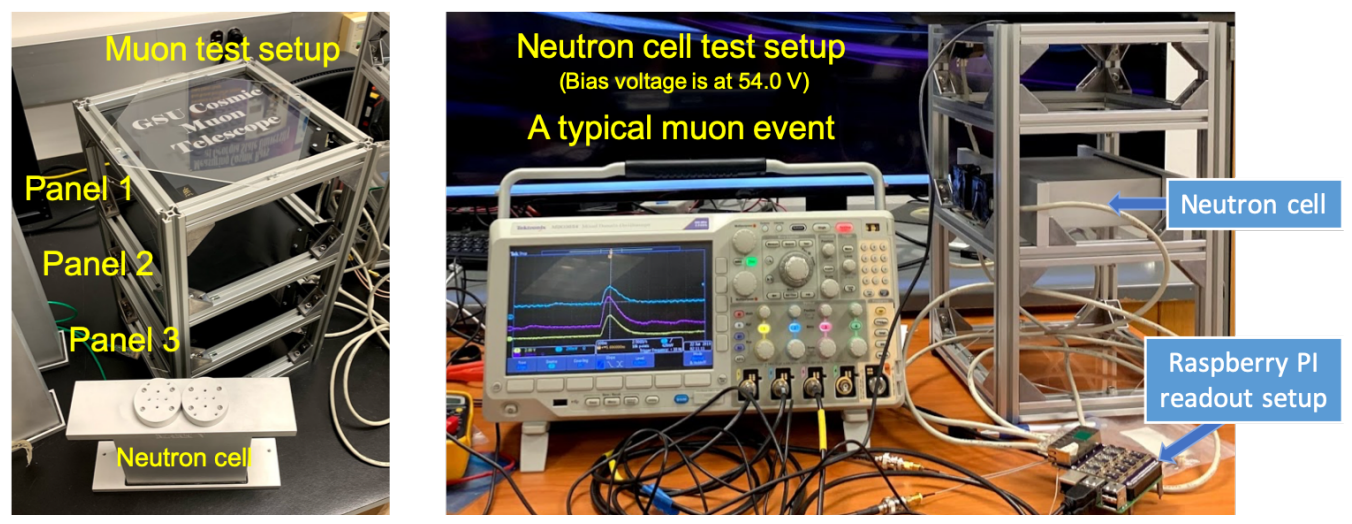

Figure 8: Cosmic ray telescope test setup. The photo on left shows the muon telescope configuration. The scope traces were recorded from the two channels of a neutron cell and one scintillator panel as seen in the photo on right. Also shown in the right photo is the Raspberry PI readout.

locations to serve as an initial exploration of monitoring cosmic ray flux variation at global scale. There are 10 units with fast neutron measurement capabilities.

Given the modular design and the easy operation of the telescope, there are a number of important applications that can be applied to with these telescopes, especially for the space and earth weather monitoring.

These telescopes can also be used as a great training tool for students at different levels. Assembling and testing of these telescopes have been an exercise both for the undergraduate and 

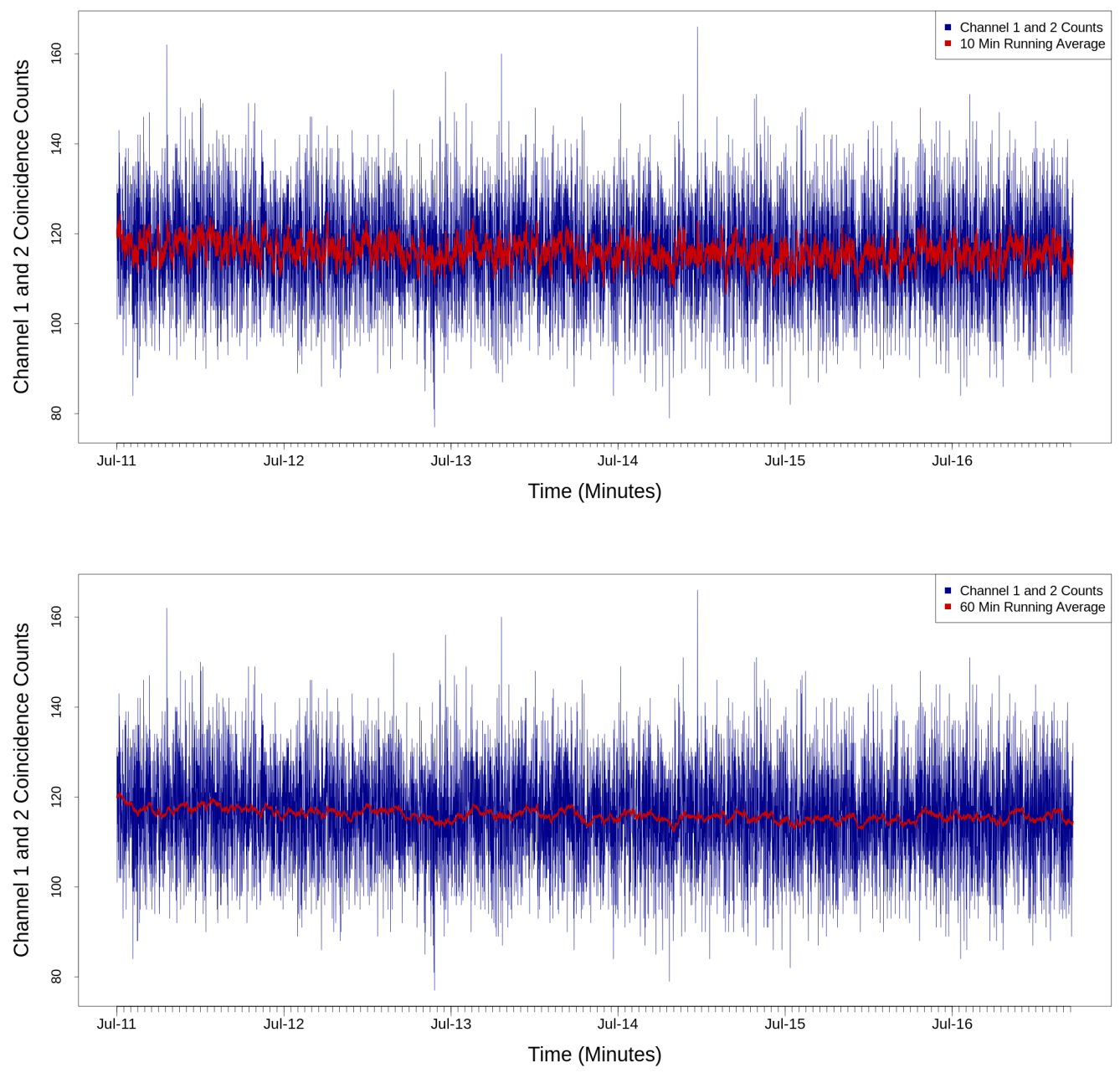

Figure 9: Coincidence count variation of the top two scintillator panels.

graduate students at Georgia State University. It provides our students with an opportunity to be innovative, to take data and to analyze data. In the summer of 2019, a rising junior high school student made great contributions by building the telescope frames and testing the detector performance.

There are a number of upgrades under consideration for the current telescope design, especially for the readout system including the SiPM bias voltage supply. The GPS unit and the environmental sensors have not been fully implemented in the available units.

On a longer term, the readout will be replaced with ASIC chip which is currently under development at Northwestern Polytechnical University, Xi'an, China. 


\section{References}

[1] Borozdin, K. N. and et al. 2003, Surveillance: Radiographic imaging with cosmic-ray muons, Nature, 422, 277.

[2] Borozdin, K. et al. 2012, Cosmic Ray Radiography of the Damaged Cores of the Fukushima Reactors, PRL, 109, 152501.

[3] Kunihiro Morishima and et al. 2017, Discovery of a big void in Khufu's Pyramid by observation of cosmic-ray muons, Nature, 552, 386-390.

[4] G. Zumerle and et al. 2018, The Cosmic Muon Tomography (CMT) Project,

http://mutomweb.pd.infn.it

[5] Jasper Kirkby, 2007, Cosmic Rays and Climate, Surv. Geophys, 28, 333-375.

[6] Lu, Q.B. 2009, Correlation between Cosmic Rays and Ozone Depletion, Phys. Rev. Lett., 102(11), 118501.

[7] A. Ollila 2012, Changes in cosmic ray fluxes improve correlation to global warming, Int. J. Phys. Scie., 7, 822-826.

[8] N. J. Shaviv 2005, On climate response to changes in the cosmic ray flux and radiative budget, J. Geophys. Res, 110, A08105.

[9] Henrik Svensmark 1998, Influence of Cosmic Rays on Earth's Climate, Phys. Rev. Lett., 81, 5027-5030.

[10] Kennedy, A. R. 2014, Biological Effects of Space Radiation and Development of Effective Countermeasures, Life Scie. Space Res., 1, 10-43.

[11] Mertens, C. J. and et al. 2013, Space Weather, 11(10), 603-635.

[12] Wilson, J.W., \& Mertens, C.J., \& Goldhagan, P., \& Copeland, K., \& Bidasaria, H. B. 2005, Atmospheric ionizing radiation and human exposure (NASA Technical Paper, NASA/TP-2005-213935, Washington, D. C.)

[13] C. A. Aidala et al., Design and Beam Test Results for the sPHENIX Electromagnetic and Hadronic Calorimeter Prototypes, in IEEE Transactions on Nuclear Science, vol. 65, no. 12, pp. 2901-2919, Dec. 2018. doi: 10.1109/TNS.2018.2879047

[14] Lewis,J. M. and Kelley,R. P. and Murer,D. and Jordan,K. A., Fission signal detection using helium-4 gas fast neutron scintillation detectors, Appl. Phys. Lett. 105, 014102 (2014); https://doi.org/10.1063/1.4887366.

[15] J. Allison, et al., Recent developments in Geant4, Nuclear Instruments and Methods in Physics Research Section A: Accelerators, Spectrometers, Detectors and Associated Equipment, Volume 835, 2016, Pages 186-225, ISSN 0168-9002, https://doi.org/10.1016/j.nima.2016.06.125.

[16] M. Tanabashi et al. (Particle Data Group), Phys. Rev. D98, 030001 (2018). 